\title{
Primary central nervous system lymphoma: is whole-body CT and FDG PET/CT for initial imaging reasonable?
}

\author{
Hana Malikova \\ Department of Radiology, Third Faculty of Medicine, Charles University and Faculty Hospital Kralovske Vinohrady, Prague, Czech Republic \\ Correspondence to: Hana Malikova. Department of Radiology, Third Faculty of Medicine, Charles University and Faculty Hospital Kralovske \\ Vinohrady, Srobarova 50, 10034 Prague, Czech Republic. Email: hana.malikova@fnkv.cz. \\ Comment on: Suh CH, Kim HS, Park JE, Jung SC, Choi CG, Kim SJ. Primary central nervous system lymphoma: Diagnostic yield of whole-body \\ CT and FDG PET/CT for initial systemic imaging. Radiology 2019;292:440-6.
}

Submitted Aug 30, 2019. Accepted for publication Sep 05, 2019.

doi: 10.21037/qims.2019.09.06

View this article at: http://dx.doi.org/10.21037/qims.2019.09.06

Central nervous system (CNS) lymphoma is an aggressive neoplasm that may involve brain, spinal cord, meninges and eyes. Primary CNS lymphoma (PCNSL) is limited only to CNS, mostly to the brain. Isolated affection of meninges, eyes or spinal cord is rare (1). However, CNS may be affected secondarily by systemic (body) lymphoma; therefore, secondary CNS lymphoma (SCNSL) was sometimes called metastatic lymphoma. PCNSL accounts for about $1 \%$ of all non-Hodgkin lymphomas (NHL) and for approximately $2 \%$ of all CNS tumors (2). The majority of PCNSL cases are diffuse large B-cell lymphomas (1). The PCNSL incidence, etiology, sex and age distribution differs significantly in immunocompetent and immunocompromised patients. Annual incidence rate of PCNSL is 7 cases per 1,000,000 people in the U.S. (3). The incidence in immunocompetent patients is increasing in the last years and PCNSL is equally common in females and males with median age at diagnosis of 67 years (1). On the contrary, the incidence in immunocompromised patients, mostly due to HIV, declined significantly during the last decades. Shiels et al. performed an incidence study, and included more than 4,000 cases, $36 \%$ of them were HIV-positive. When they compared the HIV-associated PCNSL incidence in 1990s with that between 2007-2011, there was an apparent decline from $64 \%$ of all PCNSL to $13 \%$ in the more recent period (1). Affected HIVpositive PCNSL patients were predominantly younger men with median age at diagnosis of 37 years (1). Moreover, it has been proved that PCNSL has different etiology in immunocompetent and immunocompromised patients (4). In the majority of HIV-positive and transplant recipient cases, PCNSL is associated with Epstein-Barr virus, whereas Epstein-Barr virus associated PCNSL is uncommon in immunocompetent subjects (4).

Magnetic resonance imaging (MRI) with gadoliniumbased contrast agents' applications is without doubt a powerful diagnostic modality. However, neither MRI nor histopathology is capable to distinguish between PCNSL and SCNSL $(5,6)$. Therefore, in case of histopathologically proven CNS lymphoma according to clinical oncological guidelines, contrast-enhanced chest and abdominopelvic CT or whole-body 18-fluorodeoxyglucose (FDG) positron emission tomography/computerized tomography (PET/ $\mathrm{CT}$ ) is standardly indicated to exclude the secondary brain involvement by systemic lymphoma (7).

In the August issue of Radiology 2019, the remarkable original article by Suh et al. was published (8). Authors tested the diagnostic yield of whole-body CT and FDG $\mathrm{PET} / \mathrm{CT}$ for initial systemic imaging in patients with histologically proven CNS lymphoma. They retrospectively evaluated patients, who were examined in their institution for CNS lymphoma between January 1998 and October 2018. They included 304 patients, 180 males (mean age of $58 \pm 13$ years) and 124 females (mean age of $59 \pm 13$ years), all of them underwent conclusive stereotactic biopsy. In $95 \%$ of them diffuse large B-cell lymphoma and in 1\% of them T-cell lymphoma was proven; the rest of them suffered from rare or unspecified types of lymphomas. Subsequently, 
302 patients of the whole group underwent contrastenhanced chest and abdominopelvic CT, 253 were scheduled for native whole-body FDG PET/CT and 251 underwent both diagnostic methods before therapy initiation. CT or FDG PET/CT was positive in $19(6 \%)$ subjects, these patients subsequently underwent biopsy, surgery, other invasive procedures (as cystoscopy, esophagogastroduodenoscopy) or follow-up imaging for confirmation of suspected lesions. In 13 of 19 suspected whole-body scans histopathology or following procedures proved benign lesions and excluded systemic lymphoma, therefore, those scans were false-positive. In 6 patients, the diagnosis of systemic (body) lymphoma was definitely proven, which accounts for only $2 \%$ of true-positive wholebody scans! Then Suh et al. divided subjects into several subgroups, which were separately tested for diagnostic yield of whole-body imaging and came to the following results: in patients under 65 years old the diagnostic yield of whole-body imaging was $1.4 \%$, in patients with diffuse large B-cell lymphoma $1.7 \%$ and in subjects younger than 65 years with diffuse large B-cell lymphoma was wholebody imaging true positive in 1\% (8). Authors collected imaging data from different scanners and different vendors during 20 years. Although they acknowledged technical diversity of their imaging databank, diagnostic yield of whole-body imaging scans was not significantly influenced. In patients diagnosed before 2009 diagnostic yield of wholebody scans was $2.3 \%$, while in subjects diagnosed in the last decade diagnostic yield was $1.9 \%$ (8). These findings support my feeling that technical diversity of their imaging database was not a serious limitation of the study. At last Suh $e t$ al. assessed diagnostic yield of whole-body CT and/ or PET/CT in patients with PCNSL recurrence (8). In this part of study, they included 68 patients after PCNSL therapy with suspected recurrence in the brain. In $5.5 \%$ of them whole-body imaging was false-positive and only in $1.5 \%$ of cases ( $=1$ subject) the whole-body imaging was true-positive (8). Again, diagnostic yield of the whole-body contrast-enhanced CT and/or whole-body FDG PET/CT was very low (8). The conclusion of the study by Suh et al. is straightforward: the contrast-enhanced whole-body CT and/or FDG PET/CT as an initial staging modality for suspected PCNSL should be carefully considered due to the low diagnostic yield.

The results of Suh et al. are noteworthy from several standpoints. Firstly, the study population was sufficiently large. The previous published papers, that reported significantly higher diagnostic yield of whole-body imaging
( $7.1 \%$ and $12.5 \%$, respectively), studied incomparably lower population (16 and 42 subjects, respectively) $(9,10)$. In our institution, we have been dealing with CNS lymphoma for years and according to our previous studies, in which 85 CNS lymphomas' cases were evaluated in detail during a period longer than 5 years, we found only 2 SCNSL subjects with neurological symptoms as the first manifestation of systemic lymphoma $(5,6,11)$. Thus, our data indirectly support the findings of Suh et al. Unfortunately, Suh et al. did not provide any clinical data about the clinical manifestation of CNS lymphoma. Secondly, I do not see the retrospective design of their study as very limiting factor. PCNSL is a rare disease and collecting a substantial study population takes decades for a single institution. Last but not least, I appreciate the evaluation of subjects with PCNSL recurrence, just in one patient the systemic lymphoma was proven and the rest of positive scans were false-positive and nearly four times exceeded true-positive scans (8). I am not aware of any other study, which provides data in PCNSL recurrence in sufficiently large study population.

When I am reading the study of Suh et al., I am in temptation to raise some controversial questions. Do we not waste precious time by whole-body scans for initial imaging? It was published that patients suffering either from glioblastoma or PCNSL come to the first diagnostic MRI approximately at the same time of their first neurological symptoms onset (median 30 and 25 days, respectively) (11). However, the time from the first diagnostic MRI to the first surgery or the first stereotactic biopsy was significantly longer in PCNSL patients (median 30 days in PNSCL versus 8 days in glioblastoma) (11). It is caused by morphological MRI variabilities and variability of clinical manifestation of PCNSL $(5,6)$. PCNSL brain affection may be tricky, may mimic other disease, lesions may migrate or even spontaneously disappear (12). Often glucocorticoid therapy affects both clinical symptoms and MRI findings, which leads to postponing stereotactic biopsy $(5,13)$. Moreover, the stereotactic biopsy may be nonconclusive due to glucocorticoid treatment and must be repeated, thus the time to the first stereotactic biopsy does not exactly mean the time to definitive diagnosis (13). Above listed factors may be responsible for substantial diagnosis and treatment delay. It is well known that PCNSL is a very aggressive neoplasm and its progression may be very fast. Although in the study of Suh et al. all whole-body scans were performed during one week after histologically proven diagnosis, it is still 7 days more with $2 \%$ of diagnostic 
yield. I would like to believe that it is only 7 days more; however, it is necessary to admit possibilities that FDG PET/CT scanners are not available so fast in all institutions worldwide and patients may wait longer. Next prolongation of the therapy may be caused by false-positive results of whole-body imaging that more than twice exceeded truepositive cases. The consequences of false-positive findings include unnecessary surgery, biopsy or other diagnostic invasive procedure or at least patients may wait for the follow-up scans. In case of PNCSL every day is precious and every lost day may affect the prognosis and outcome of the single patient. We can harm PCNSL patients not only by therapy delay but also by the unnecessary procedures and their side-effects.

In the last years the question of radiation exposure has been increasingly concerned. From 304 included patients, 251 of them underwent both contrast-enhanced wholebody CT and native whole-body FDG PET/CT scans. Why both methods had been used? Is this approach really reasonable? Does this approach meet the As Low As Reasonable Achievable (ALARA) principles? What is the real influence of true-positive whole-body scans on the patients' treatment? Recently, the term "defensive medicine" is often used for overusing diagnostic procedures that leads not only to unnecessary radiation exposure of patients, but also to inadequately increasing medical costs.

The article of Suh et al. leads my thoughts to the established clinical protocols. The study of Suh et al. might show us only a "tip of the iceberg". Are the clinical guidelines and established protocols really based on recent studies and latest scientific results? Certainly, clinical guidelines should not be changed according to a single study, it should be done on the base of meta-analysis or systematic review. However, a single study may show pitfalls of established clinical protocols. According to my opinion the study by Suh et al. should be replicated; an ideal design for the next study should be multicenter and prospective; however, it may be difficult in reality. If the results of Suh et al. were supported by the next research with adequate size of included populations and used methods, then would be time for considering of critical revision of the clinical guidelines.

In my commentary I put forward several questions, most of them controversial. It was not my intention to answer all of them, it is not possible in these days. However, we can look for the ways how to solve them.

\section{Acknowledgments}

$\mathrm{H}$ Malikova is supported by Charles University research program PROGRES Q28.

\section{Footnote}

Conflicts of Interest: The author has no conflicts of interest to declare.

\section{References}

1. Shiels MS, Pfeiffer RM, Besson C, Clarke CA, Morton LM, Nogueira L, Pawlish K, Yanik EL, Suneja G, Engels EA. Trends in primary central nervous system lymphoma incidence and survival in the U.S. Br J Haematol 2016;174:417-24.

2. Ostrom QT, Gittleman H, de Blank PM, Finlay JL, Gurney JG, McKean-Cowdin R, Stearns DS, Wolff JE, Liu M, Wolinsky Y, Kruchko C, Barnholtz-Sloan JS. American Brain Tumor Association Adolescent and Young Adult Primary Brain and Central Nervous System Tumors Diagnosed in the United States in 2008-2012. Neuro Oncol 2016;18:11-50.

3. O'Neill BP, Decker PA, Tieu C, Cerhan JR. The changing incidence of primary central nervous system lymphoma is driven primarily by the changing incidence in young and middle-aged men and differs from time trends in systemic diffuse large B-cells non-Hodgkin's lymphoma. Am J Hematol 2013;88:997-1000.

4. Bibas M, Antinori A. EBV and HIV-Related Lymphoma. Mediterr J Hematol Infect Dis 2009;1:e2009032.

5. Koubska E, Weichet J, Malikova H: Central nervous system lymphoma: a morfological MRI study. Neuro Endocrinol Lett 2016;37:318-24.

6. Malikova H, Burghardtova M, Koubska E, Mandys V, Kozak T, Weichet J. Secondary Central Nervous System Lymphoma: Spectrum of Morphological MRI Appearances. Neuropsychiatr Dis Treat 2018;14:733-40.

7. National Comprehensive Cancer Network. Clinical practice guidelines in oncology for central nervous system cancers V.1.2018. 2018. Available online: http://www.nccn. org. Accessed August 2019.

8. Suh CH, Kim HS, Park JE, Jung SC, Choi CG, Kim SJ. Primary Central Nervous System Lymphoma: Diagnostic Yield of Whole-Body CT and FDG PET/CT for Initial 
Systemic Imaging. Radiology 2019;292:440-6.

9. Ferreri AJ, Reni M, Zoldan MC, Terreni MR, Villa E. Importance of complete staging in non-Hodgkin's lymphoma presenting as a cerebral mass lesion. Cancer 1996;77:827-33.

10. Mohile NA, Deangelis LM, Abrey LE. The utility of body FDG PET in staging primary central nervous system lymphoma. Neuro Oncol 2008;10:223-8.

11. Malikova H, Koubska E, Weichet J, Klener J, Rulseh A, Liscak R, Vojtech Z. Can morphological MRI differenciate between primary central nervous system lymphoma and glioblastoma? Cancer Imaging 2016:16:40.

12. Al-Yamany M, Lozano A, Nag S, Laperriere N, Bernstein M. Spontaneous remission of primary central nervous system lymphoma: report of 3 cases and discussion of pathophysiology. J Neurooncol 1999;42:151-9.

13. Malikova H, Liscak R, Latnerova I, Guseynova K, Syrucek M, Pytlik R. Complications of MRI-guided stereotactic biopsy of brain lymphoma. Neuro Endocrinol Lett 2014;35:613-8.

Cite this article as: Malikova $\mathrm{H}$. Primary central nervous system lymphoma: is whole-body CT and FDG PET/CT for initial imaging reasonable? Quant Imaging Med Surg 2019;9(9):1615-1618. doi: 10.21037/qims.2019.09.06 